\title{
Perfil epidemiológico da tuberculose óssea no Brasil, 2001-2017
}

\author{
Epidemiological profile of bone tuberculosis in Brazil, 2001-2017
}

\section{Ricardo Vieira Teles Filho ${ }^{1}$, Guilherme de Matos Abe ${ }^{1}$, Lucas Henrique Souza de Azevêdo ${ }^{1}$, Nilo Carrijo Melo², Marcelo Fouad Rabahi ${ }^{3}$, Murilo Tavares Daher ${ }^{1,2}$}

Teles Filho RV, Abe GM, Azevêdo LHS, Melo NC, Rabahi MF, Daher MT. Perfil epidemiológico da tuberculose óssea no Brasil, 201-2017 / Epidemiological profile of bone tuberculosis in Brazil, 2001-2017. Rev Med (São Paulo). 2019 set.-out.;98(5):315-23.

RESUMO: Objetivo: Delinear o perfil epidemiológico da tuberculose óssea (TBO) no Brasil no período de 2001 a 2017. Métodos: Estudo epidemiológico retrospectivo com abordagem descritiva e quantitativa. Os dados foram obtidos no Sistema de Informação de Agravos de Notificação (SINAN), disponível para consulta no banco de dados do Departamento de Informática do Sistema Único de Saúde (DATASUS). Resultados: Houve redução do número de casos de tuberculose se comparados os números absolutos de 2001 e 2017, e que o acometimento ósseo permanece prevalente, sendo a quinta causa de acometimento sabidamente extrapulmonar no Brasil. Constatou-se que na maioria dos casos, os indivíduos são do sexo masculino, com idade entre 20 a 59 anos, que residem em áreas urbanas, no Sudeste do país. Conclusão: A TBO continua sendo um importante foco de envolvimento extrapulmonar no Brasil, principalmente nos grupos economicamente ativos onde é mais prevalente.

Descritores: Tuberculose; Tuberculose osteoarticular; Epidemiologia; Tuberculose da coluna vertebral; Brasil/ epidemiologia

\begin{abstract}
Objective: This study aimed to delineate the epidemiological profile of bone tuberculosis (BT) in Brazil from 2001 to 2017. Methods: Retrospective study with a descriptive and quantitative approach. Data was obtained from the Sistema de Informação de Agravos de Notificação (SINAN) available for consultation in the database of the Departamento de Informática do Sistema Único de Saúde (DATASUS). Results: There was reduction in the number of cases of tuberculosis when comparing the whole numbers of 2001 and 2017, and the bone involvement remains prevalent, being the fifth cause of extrapulmonary involvement in Brazil. In most cases, the individuals are male, aged between 20-59 years, living in urban areas, in the Southeast of the country. Conclusion: BT remains an important focus of extrapulmonary involvement in Brazil, and affects mainly the economically active groups.
\end{abstract}

Keywords: Tuberculosis; Tuberculosis, osteoarticular; Epidemiology; Tuberculosis, spinal; Brazil/Epidemiology.

1. Universidade Federal de Goiás (UFG), Departamento de Ortopedia e Traumatologia DOT/FM/UFG, Goiânia, GO. ORCID: Teles Filho RV - https:// orcid.org/0000-0003-4822-1526; Abe GM - https://orcid.org/0000-0002-2446-3750; Azevêdo LHS - http://orcid.org/0000-0002-2629-492X. Email: ricardovteles@gmail.com, guimatosabe@gmail.com, lucasvedo42@gmail.com.

2. Centro de Reabilitação e Readaptação Dr. Henrique Santillo - CRER, Goiânia, GO. ORCID: Melo NC - http://orcid.org/0000-0003-3879-6991; Daher MT - http://orcid.org/0000-0001-9589-5596.Email: drnilocarrijo@gmail.com; murilodaher@uol.com.br.

3. Universidade Federal de Goiás (UFG), Departamento de Clínica DCM/FM/UFG, Goiânia, GO. ORCID: http://orcid.org/0000-0002-4050-5906. Email:mfrabahi@gmail.com.

Endereço para correspondência: Ricardo Vieira Teles Filho. Avenida dos Alpes, Quadra 49, Lote 11, Setor União. Goiânia, GO. CEP: 74313-760. E-mail: ricardovteles@gmail.com. 


\section{INTRODUÇÃO}

tuberculose (TB) é grave problema de saúde pública no mundo. Em 2016, 10,4 milhões de pessoas contraíram a doença e cerca de 1,3 milhões morreram em decorrência dela. Segundo a Organização Mundial da Saúde, é a doença infecciosa de agente único que mais mata, superando a $\operatorname{AIDS}^{1}$. No Brasil foram registrados 69.569 casos novos em 2017 e 4.426 óbitos por TB em $2016^{2}$. Os coeficientes de incidência e de mortalidade por TB apresentaram redução média anual de 1,6\% (2008 a 2017) e 2,0\% (2007 a 2016), respectivamente ${ }^{3}$.

Sabe-se que em casos de TB, a forma pulmonar é a mais frequente. $\mathrm{O}$ acometimento extrapulmonar representa cerca de $10 \%$ dos casos, e eles são classificados segundo sua localização: laríngea, ganglionar periférica, meningoencefálica, óssea, genitourinária, miliar, cutânea e ocular ${ }^{4}$. Deste total, $50 \%$ estão relacionados ao sistema musculoesquelético. A TB óssea (TBO) acomete mais comumente a coluna vertebral, a articulação coxofemoral e o joelho, embora possa ocorrer em vários outros locais ${ }^{5,6}$. A TB espinhal (TBE), ou mal de Pott, corresponde a 1 a $2 \%$ dos casos de TB extrapulmonar (TBEx) $)^{7,8}$, sendo afetados mais comumente os segmentos torácico baixo e lombar 9 .

De modo geral, o quadro infeccioso ósseo se manifesta como artrite e/ou osteomielite, havendo inflamação e/ou infecção articular. Na coluna vertebral, acomete as vértebras e discos adjacentes. Apresenta progressão indolente se comparada às osteomielites e pioartrites inespecíficas, porém exibe grande morbidade ${ }^{10,11}$. Dentre os fatores de risco associados ao desenvolvimento da TBO, alguns têm destaque epidemiológico, como localização geográfica, faixa etária, tratamento com imunossupressores, diabetes mellitus e infecção por HIV ${ }^{5}$.

Tendo em vista a alta prevalência da TB no Brasil e a grande importância do acometimento ósseo, que deve ser prevenido e controlado por meio de medidas direcionadas para cada segmento social, seja conforme o sexo, idade ou condições socioeconômicas, com abordagens diferentes de acordo com as particularidades existentes relacionadas às realidades de cada região, este trabalho tem por objetivo delinear o perfil epidemiológico da TBO no Brasil.

\section{MÉTODOS}

Trata-se de estudo retrospectivo com abordagem descritiva e quantitativa. Os dados foram obtidos no DATASUS entre 14/09/2018 e 14/10/2018, que utiliza como fonte de informação o SINAN, para registro dos casos de TB, cuja notificação é compulsória ${ }^{12}$. Trata-se de casos confirmados no período de 2001 a 2017.

Oito variáveis foram selecionadas para análise: sexo, etnia, faixa etária, zona de residência, região geográfica, condições socioeconômicas, comorbidades e uso de drogas. Os dados foram analisados descritivamente e agrupados em planilha do Software Microsoft Excel ${ }^{\circledR}$.

Em razão de este estudo agregar dados secundários disponíveis em base de dados governamental de domínio público e não envolver diretamente seres humanos e sua possível identificação, a submissão do projeto de pesquisa ao Comitê de Ética de nossa Instituição foi dispensada, porém foram seguidas as demais normas relativas a este (CNS: 466/12).

\section{RESULTADOS}

\section{Distribuição dos casos de tuberculose extrapulmonar no Brasil de 2001-2017}

Em 2001, dos 87.265 casos de TB, 15.614 (17,89\%) foram classificados como TBEx, e, destes, 683 como TBO, o que corresponde a $4,4 \%$ dos casos de TBEx - colocando-a na $6^{\mathrm{a}}$ posição entre as formas de TBEx (Tabela 1). Em 2017, dos 89.487 casos de TB, 13.457 $(15,03 \%)$ foram classificados como TBEx, e, destes, 639 como TBO, correspondendo a 4,75\% dos casos de TBEx - colocando-a, agora, na $5^{\text {a }}$ posição entre as formas de TBEx. Assim, embora tenha havido um aumento de cerca de $2,55 \%$ no número total de casos totais de TB no período, o número de casos de TBO decresceu cerca de 6,44\%. Evidencia-se, a partir desses dados, tendência à diminuição de ambas as variáveis, apesar do aumento do número absoluto, conforme evidenciado pelas linhas de tendência do Gráfico 1.

O número de casos totais de TB em um ano atingiu seu máximo em 2003 (93.773 casos) e seu mínimo em 2014 (84.843 casos). Essas variações não foram, entretanto, acompanhadas pelos casos totais de TBO, que atingiram seu máximo em 2002 (776 casos) e seu mínimo em 2010 (608 casos). 
Tabela 1. Número de diagnósticos de TB extrapulmonar por ano do diagnóstico

\begin{tabular}{ccccccccccc}
\hline $\begin{array}{c}\text { Ano } \\
\text { diagnóstico }\end{array}$ & Pleural & Ganglionar Periférica & Genitourinária & Óssea & Ocular & Miliar & Meningo-encefálica & Cutânea & Laríngea & Outra \\
\hline $\mathbf{2 0 0 1}$ & 0 & 3134 & 522 & 683 & 266 & 853 & 721 & 27 & 1468 & 7940 \\
$\mathbf{2 0 0 2}$ & 0 & 3290 & 650 & 776 & 325 & 918 & 727 & 37 & 1959 & 8646 \\
$\mathbf{2 0 0 3}$ & 0 & 3356 & 593 & 664 & 363 & 937 & 721 & 43 & 2238 & 8708 \\
$\mathbf{2 0 0 4}$ & 0 & 3288 & 535 & 652 & 381 & 1031 & 700 & 46 & 2270 & 8622 \\
$\mathbf{2 0 0 5}$ & 0 & 3353 & 483 & 709 & 343 & 1080 & 712 & 68 & 2007 & 8378 \\
$\mathbf{2 0 0 6}$ & 0 & 3043 & 493 & 637 & 324 & 1072 & 696 & 118 & 588 & 7620 \\
$\mathbf{2 0 0 7}$ & 3 & 3114 & 412 & 685 & 321 & 1165 & 816 & 343 & 222 & 7310 \\
$\mathbf{2 0 0 8}$ & 5 & 3203 & 375 & 701 & 362 & 1159 & 798 & 322 & 222 & 7618 \\
$\mathbf{2 0 0 9}$ & 5 & 3000 & 347 & 672 & 329 & 1119 & 805 & 316 & 201 & 7437 \\
$\mathbf{2 0 1 0}$ & 1 & 2917 & 315 & 608 & 376 & 1130 & 773 & 277 & 200 & 7202 \\
$\mathbf{2 0 1 1}$ & 7 & 2950 & 329 & 641 & 352 & 1201 & 813 & 305 & 194 & 7649 \\
$\mathbf{2 0 1 2}$ & 5 & 2838 & 330 & 670 & 346 & 1205 & 819 & 243 & 197 & 7400 \\
$\mathbf{2 0 1 3}$ & 8 & 2818 & 313 & 628 & 374 & 1203 & 862 & 236 & 175 & 7167 \\
$\mathbf{2 0 1 4}$ & 14 & 2605 & 279 & 677 & 378 & 1211 & 844 & 239 & 168 & 6839 \\
$\mathbf{2 0 1 5}$ & 0 & 2569 & 271 & 662 & 328 & 1196 & 858 & 200 & 152 & 6826 \\
$\mathbf{2 0 1 6}$ & 0 & 2709 & 256 & 616 & 369 & 1239 & 787 & 241 & 179 & 6964 \\
$\mathbf{2 0 1 7}$ & 0 & 2678 & 230 & 639 & 421 & 1166 & 888 & 222 & 164 & 7049 \\
\hline
\end{tabular}

Gráfico 1. Comparação entre a distribuição anual dos casos totais de TB e dos casos de TBO, entre 2001 e 2017, em números absolutos e em linhas de tendência

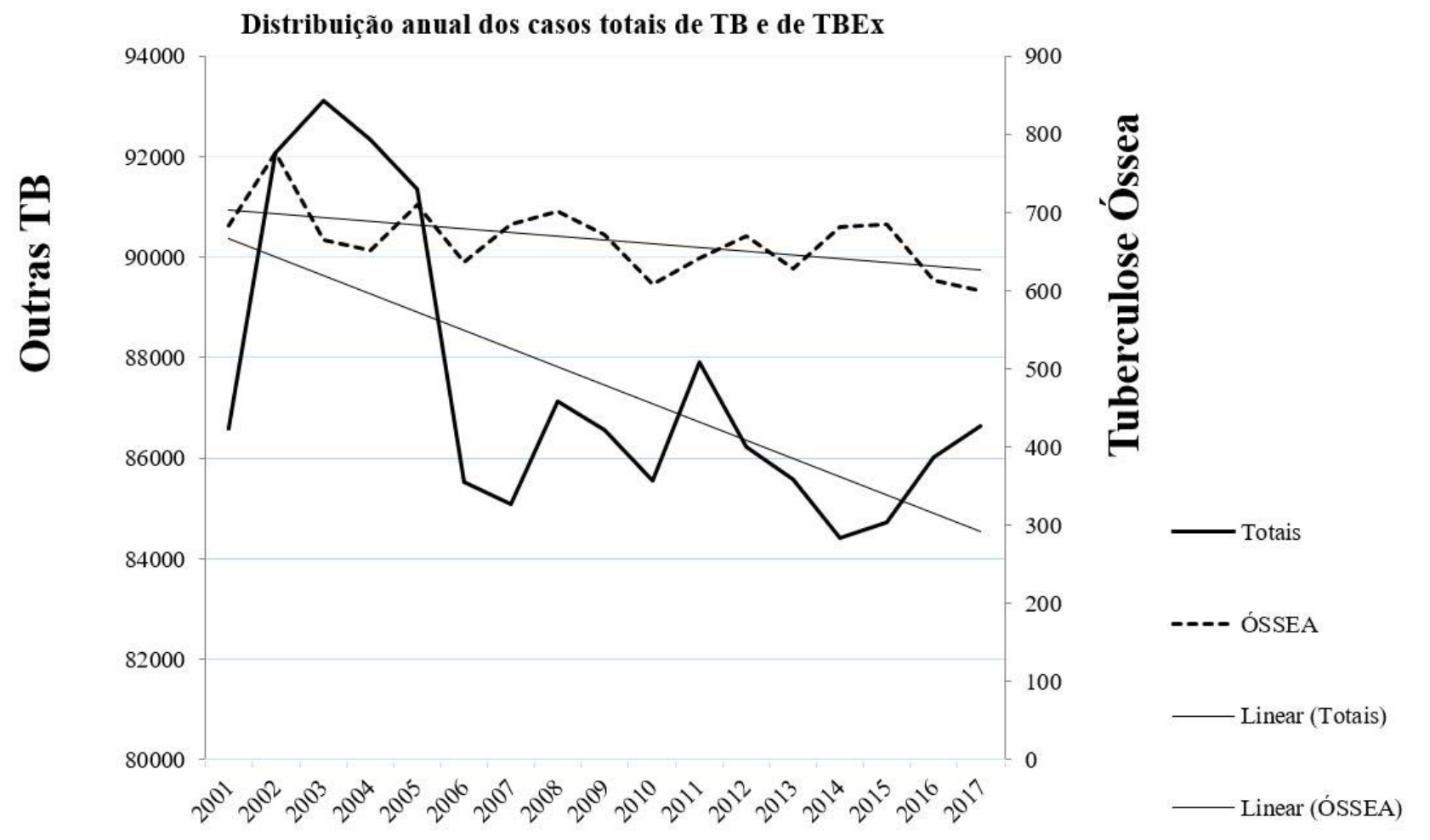




\section{Quanto ao sexo dos pacientes com diagnóstico de TBO}

Em 2001, 420 casos de TBO foram diagnosticados em pacientes do sexo masculino, o que correspondia a $61,5 \%$ do total, e 263 casos de TBO foram diagnosticados em pacientes do sexo feminino, o que correspondia a $38,5 \%$ do total. Em 2017, foram registrados, novamente, 420 casos, o que correspondia a $65,73 \%$ do total enquanto 219 casos femininos, o que correspondia a $34,28 \%$ do total.

\section{Quanto à etnia dos pacientes com diagnóstico de TBO}

É importante ressaltar que, em 2001, a grande maioria dos diagnósticos de TBO não foi registrada com a etnia correspondente do paciente: 608 casos, o correspondente a $89 \%$ do total. Em 2017, reduziu-se para 60 , o correspondente a $9,4 \%$ do total.

Em 2001, 37 casos de TBO foram diagnosticados em pacientes brancos, o que correspondia a $5,42 \%$ do total. Em 2017, foram registrados 249 casos, o que correspondia a $39 \%$ do total.

Em 2001, 35 casos de TBO foram diagnosticados em pacientes pretos ou pardos, o que correspondia a 5,12\% do total. Em 2017, foram registrados 322 casos, o que correspondia a $50,4 \%$ do total.

Em 2001, apenas 3 casos de TBO foram diagnosticados em pacientes indígenas, o que correspondia a apenas $0,44 \%$ do total. Em 2017, foram registrados apenas 4 casos, o que correspondia a apenas $0,63 \%$ do total.

\section{Quanto à idade dos pacientes com diagnóstico de TBO}

Em 2001, 111 casos de TBO foram diagnosticados em pacientes com 19 anos ou menos, o que correspondia a $16,3 \%$ do total de casos de TBO. Em 2017, foram registrados 45 casos (uma redução de 59,5\%), o que correspondia a $7,04 \%$ do total.

Em 2001, 425 casos de TBO foram diagnosticados em pacientes com idade entre 20 e 59 anos, o que correspondia a $62,23 \%$ do total de casos de TBO. Em 2017, foram registrados 429 casos, o que correspondia a $67,14 \%$ do total.

Em 2001, 146 casos de TBO foram diagnosticados em pacientes com 60 anos ou mais, o que correspondia a 21,4\% do total de casos de TBO. Em 2017, foram registrados 164 casos, o que correspondia a $25,7 \%$ do total (Gráfico 2).

\section{Quanto à zona de residência dos casos confirmados}

Em 2001, 458 casos de TBO estavam em zona urbana de residência, o correspondente a $67 \%$ do total. Em 2017, 429 casos de TBO estavam nessa zona, o correspondente também a cerca de $67 \%$ do total, e dos quais 141 casos estavam na Região Nordeste, e apenas 34 na Região Centro-Oeste. Comparando com a zona rural em 2001, 40 casos de TBO estavam em zona rural de residência, o correspondente a cerca de $6 \%$ do total. Em 2017, 43 casos estavam nessa zona, o correspondente a cerca de $6,7 \%$ do total, e dos quais 25 casos estavam na Região Nordeste, e um único caso na Região Centro-Oeste.

2001
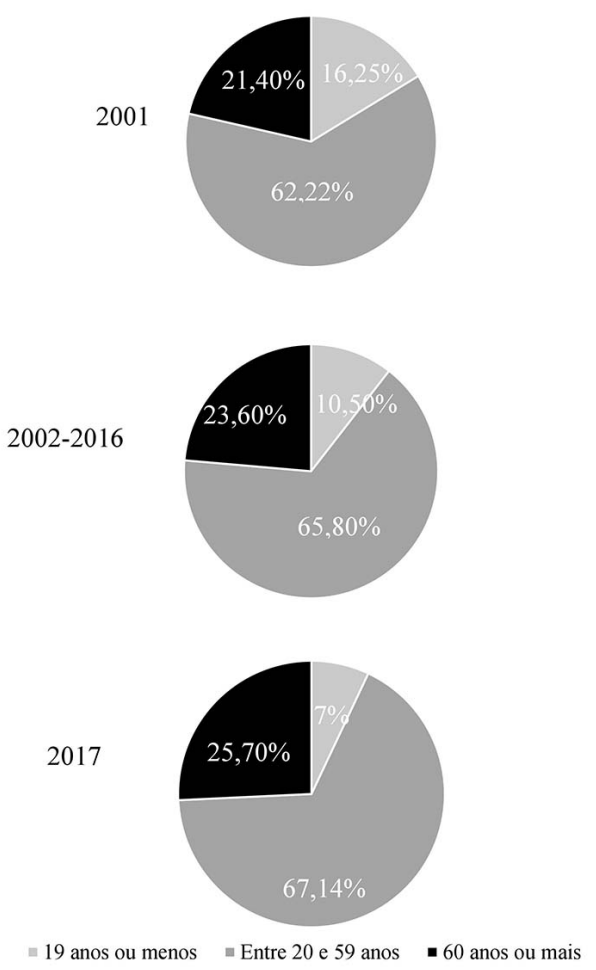

Gráfico 2. Distribuição de TBO por faixa etária

\section{Epidemiologia regional da TBO}

A Região Norte foi a que apresentou maior aumento percentual da quantidade de casos totais de TB e de TBO comparando-se 2001 e 2017: em 2001, foram diagnosticados 7.582 casos totais de TB, dos quais 37 foram classificados como TBO. Em 2017, a quantidade total de casos na região aumentou em 33\%, para 10.109 casos, e os casos de TBO aumentaram em 59,6\%, para 59 casos. Com isso, a Região Norte respondeu, em 2017, por 9,23\% do total de casos de TBO no Brasil.

A Região Nordeste foi a que apresentou maior redução percentual da quantidade de casos totais de TB comparando-se 2001 e 2017: em 2001, foram diagnosticados 25.602 casos totais de TB, dos quais 191 foram classificados como TBO. Em 2017, a quantidade total de casos na região reduziu em 7,6\%, para 23.660 casos, e os casos de TBO reduziram em $11,52 \%$, para 169 casos. Com isso, a Região Nordeste respondeu, em 2017, por $26,45 \%$ do total de casos de TBO no Brasil.

A Região Sudeste concentra a maior parte dos casos de TBO no país, atualmente. Em 2001, foram diagnosticados 39.740 casos totais de TB, dos quais 287 foram classificados como TBO. Em 2017, a quantidade total de casos na região aumentou em 2,37\%, para 40.682 
casos, e os casos de TBO aumentaram em 2,1\%, para 293 casos. Com isso, a Região Sudeste respondeu, em 2017, por $45,85 \%$ do total de casos de TBO no Brasil.

Na Região Sul, em 2001, foram diagnosticados 10.150 casos totais de TB, dos quais 99 foram classificados como TBO. Em 2017, a quantidade total de casos na região aumentou em 7,13\%, para 10.874 casos, e os casos de TBO reduziram em 16,16\%, para 83 casos. Com isso, a Região Sul respondeu, em 2017, por $13 \%$ do total de casos de TBO no Brasil.
A Região Centro-Oeste é a que possui a menor parte dos casos de TBO no país, atualmente, e foi a região que apresentou a maior redução percentual da quantidade de casos de TBO comparando-se 2001 e 2017. Na Região Centro-Oeste, em 2001, foram diagnosticados 4.191 casos totais de TB, dos quais 69 foram classificados como TBO. Em 2017, a quantidade total de casos na região reduziu em $0,70 \%$, para 4.162 casos, e os casos de TBO reduziram em 49,3\%, para 35 casos. Com isso, a Região Centro-Oeste respondeu, em 2017, por 5,5\% do total de casos de TBO no Brasil (Tabela 2).

Tabela 2. Distribuição geográfica dos casos de TB e TBO entre 2001 e 2017

\begin{tabular}{cccccc}
\hline & & $\mathbf{2 0 0 1}$ & $\mathbf{2 0 0 2 - 2 0 1 6}$ & $\mathbf{2 0 1 7}$ & Variação (2001-2017) \\
\hline Norte & TB & 7582 & 125765 & 10109 & $33 \%$ \\
& TBO & 37 & 770 & 59 & $59,56 \%$ \\
\hline Nordeste & TB & 25602 & 376123 & 23660 & $-7,60 \%$ \\
\hline Sudeste & TBO & 191 & 2833 & 169 & $-11,52 \%$ \\
& TB & 39740 & 597703 & 40682 & $2,40 \%$ \\
\hline $\begin{array}{c}\text { Sul } \\
\text { Centro }\end{array}$ & TBO & 287 & 4358 & 293 & $2,10 \%$ \\
\hline & TB & 10150 & 163332 & 10874 & $7,13 \%$ \\
\hline & TB & 99 & 1423 & 83 & $-16,20 \%$ \\
\hline
\end{tabular}

\section{Quanto às condições socioeconômicas dos pacientes}

Em 2017, foram diagnosticados apenas 8 casos de TBO em pacientes em situação de rua, o correspondente a $1,2 \%$ do total. $O$ registro desse tipo de condição foi largamente ignorado até 2014.

Em 2017, 29 casos de TBO foram diagnosticados em pacientes que recebiam algum tipo de benefício governamental, o correspondente a $4,54 \%$ do total. $\mathrm{O}$ registro desse tipo de condição foi largamente ignorado até 2016.

Em 2017, 16 casos de TBO foram diagnosticados em pacientes profissionais de saúde, o correspondente a $2,5 \%$ do total. O registro desse tipo de condição foi largamente ignorado até 2015.

Em 2017, 2 casos de TBO foram diagnosticados em pacientes imigrantes no Brasil, o correspondente a 0,3\% do total. O registro desse tipo de condição foi largamente ignorado até 2015 .

\section{Quanto aos diagnósticos de TBO em pacientes com comorbidades}

Em 2001, 45 casos de TBO eram HIV positivos, o correspondente a $6,6 \%$ do total. Neste ano, entretanto, a maior parte dos casos não foi testada para HIV: 446 casos, o correspondente a $65,4 \%$ do total. Em 2017, 95 dos casos de TBO eram HIV positivos, o correspondente a $15 \%$ do total. Neste ano, 132 casos não foram testados para HIV, o correspondente a $21 \%$ do total.

Em 2005, dos dois óbitos por TB de pacientes com TBO, um deles foi testado para HIV, e resultou HIV positivo. Em 2017, dos 15 óbitos por TB de pacientes com TBO, 11 foram testados para HIV, e nenhum deles foi HIV positivo (Gráfico 3 e 4). 
Gráfico 3. Casos de TBO e HIV por ano

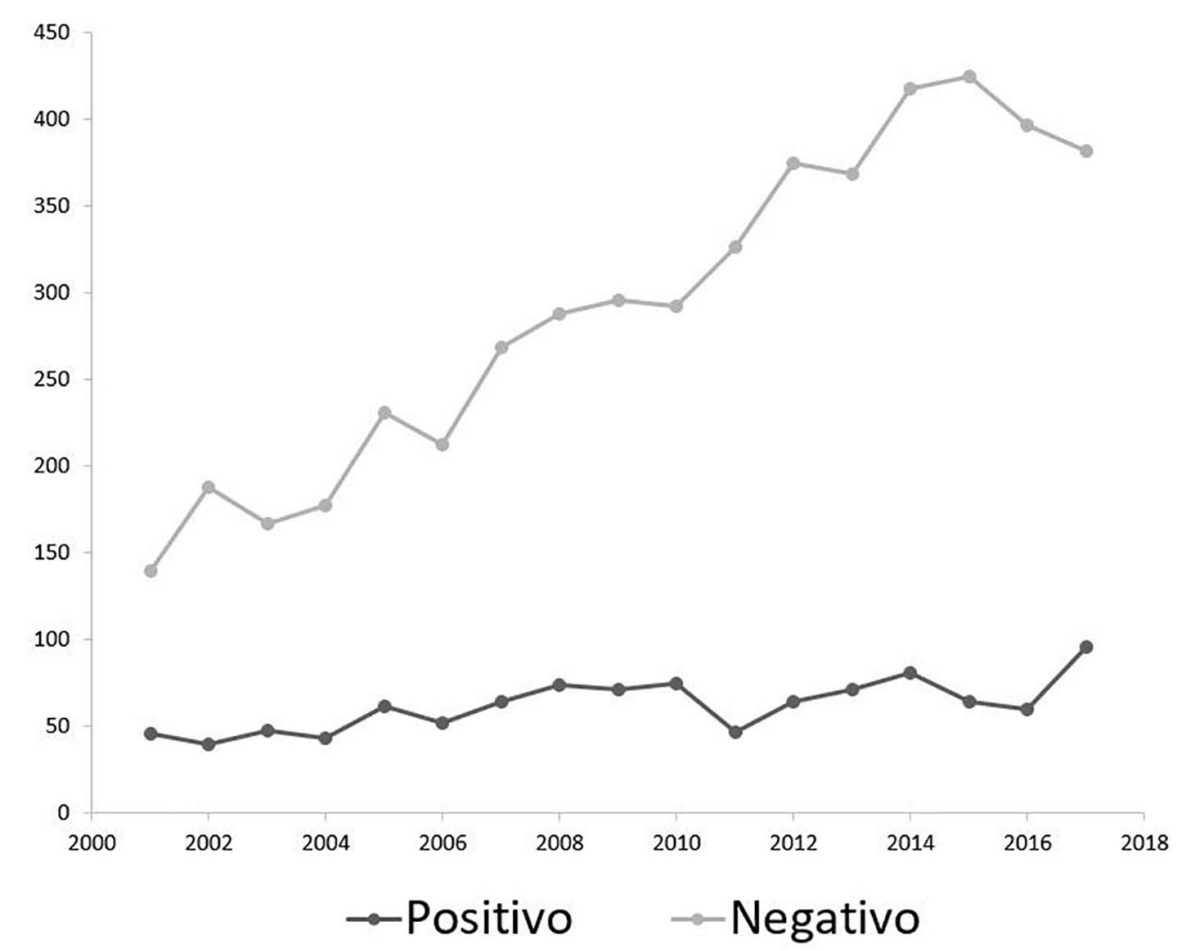

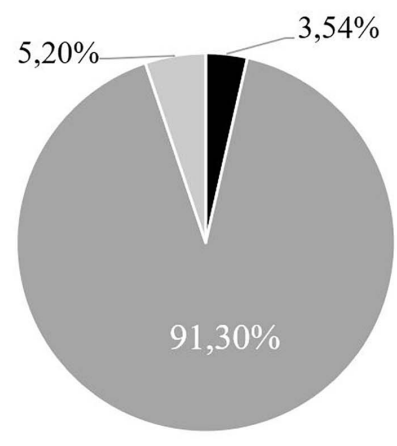

- menor de 19 anos $\square 20$ a 59 anos $\square$ maior de 60 anos

Gráfico 4. Casos de TBO e HIV distribuídos por faixas etárias

Em 2001, apenas três casos de TBO eram de pacientes diabéticos, o correspondente a $0,44 \%$ do total. Neste ano, entretanto, na maior parte dos casos, o registro de paciente diabético foi ignorado ou deixado em branco: 677 casos, o correspondente a $99,12 \%$ do total. Em 2017, 69 casos de TBO eram de pacientes diabéticos, o correspondente a $11 \%$ do total. Neste ano, em 29 casos o registro de paciente diabético foi ignorado ou deixado em branco, o correspondente a $4,54 \%$ do total.

\section{Quanto ao uso de drogas}

Em 2017, 53 casos de TBO foram diagnosticados em pacientes alcoólatras, o correspondente a $12 \%$ do total.
O registro desse tipo de condição foi largamente ignorado até 2007.

Em 2017, 68 casos de TBO foram diagnosticados em pacientes tabagistas, o correspondente a $10,6 \%$ do total. $\mathrm{O}$ registro desse tipo de condição foi largamente ignorado até 2015.

Em 2017, 26 casos de TBO foram diagnosticados em pacientes usuários de drogas ilícitas, o correspondente a $4 \%$ do total. O registro desse tipo de condição foi largamente ignorado até 2015.

Portanto, o perfil epidemiológico da TBO no Brasil revela que esta representa $4,75 \%$ dos casos de TBEx no Brasil, o correspondente a $0,7 \%$ dos casos totais de TB, com tendência à diminuição; no entanto, mais lenta do que a de casos de TB. É uma afecção predominante em homens brancos, entre 20 e 59 anos, e residentes em áreas urbanas da região Sudeste do país. Estes dados foram obtidos de dados do DATASUS em que há grande número de ignorados no período, comprometendo a análise das variáveis relativas a comorbidade e uso de drogas. Sendo assim, os resultados talvez não demonstrem o real estado desta condição.

\section{DISCUSSÃO}

Os casos de TBO representam cerca de 2 a 3\% de todos os casos de TB notificados nos Estados Unidos, valores maiores do que os encontrados neste estudo (0,7\%); o mesmo ocorre para a parcela de casos de TBO entre os casos totais de TBEx, que é de $4,75 \%$ no Brasil, conforme 
nossos dados, e 9,6\% nos EUA ${ }^{13}$. Nosso estudo também identificou que $15,03 \%$ dos casos totais de TB no Brasil são de TBEx, valores superiores aos previamente reportados, de $10 \%$ em média ${ }^{4}$, porém inferiores à estatística americana, de $20,2 \%{ }^{13}$. Há de se notar que, ao contrário do Brasil, naquele país a doença não é endêmica. Em algumas séries relatadas no Reino Unido, mais de $6 \%$ dos casos de TB foram causados por doença óssea e articular, proporções inferiores às brasileiras, apesar da disparidade no número total de casos de TB. Nesses países tem sido relatada diferença racial crescente nos últimos anos, com a maioria dos casos acometendo minorias e nascidos fora do país ${ }^{13,14}$. Esses imigrantes foram responsáveis pelo aumento da incidência da doença nesses países. Em razão do comportamento indolente da doença e baixa suspeita clínica em áreas com baixa incidência de TB, o atraso no diagnóstico é frequente nos países desenvolvidos ${ }^{15,16}$.

Nos países onde a tuberculose é endêmica, crianças mais velhas e adultos jovens são mais comumente afetados com $\mathrm{TBO}$, o que corresponde à realidade brasileira, em que a grande maioria dos casos se encontra após a $3^{\mathrm{a}}$ década de vida, enquanto nos países desenvolvidos a doença é frequentemente observada em idosos ${ }^{17}$. Historicamente, a TBO foi uma doença de crianças e adolescentes, muitas vezes vista se desenvolvendo dentro de alguns anos após infecção primária. Em áreas com recursos limitados, ainda é frequente, enquanto nos países desenvolvidos a TBO resulta mais comumente de reativações ${ }^{18,19}$. Em uma série de 194 pacientes da Índia com TBO, 30\% dos casos ocorreram durante a $2^{\mathrm{a}}$ década de vida, $22 \%$ na $1^{\mathrm{a}}$ década, $18 \%$ na $3^{\mathrm{a}}$ década e $14 \%$ na $4^{\text {a }}$ década $^{20}$, semelhante ao nosso quadro nacional, com exceção do nosso declínio dos casos antes da $2^{\circ}$ década e do nosso alto índice após a $6^{\circ}$ década.

A TB vertebral é o tipo mais comum de TBO tanto em países endêmicos como não endêmicos, responsável por cerca de $50 \%$ dos casos na maioria das séries. Os dados disponíveis em nosso país utilizados para essa análise não contemplam a discriminação de sítios anatômicos da TBO, como o fazem os estudos analisados a seguir. Em estudo realizado em Los Angeles, EUA, um total de 220 casos de TBO foram registrados entre 1990 e 1995; a distribuição de sítios foi: 118 (54\%) tinham TB vertebral e 56 (26\%) tinham envolvimento articular (29 casos (13\%) com envolvimento do joelho, 18 (8\%) com envolvimento do quadril e 9 (4\%) com envolvimento do punho) $)^{6,18,21}$.

Na mesma série com 194 casos na Índia de notou-se a seguinte distribuição: 49\% tinham doença vertebral, 34 (18\%) tinham envolvimento do joelho, 32 (16\%) tinham envolvimento do quadril, $15(8 \%)$ tinham envolvimento do tornozelo / pé, 8 (4\%) tinham envolvimento do cotovelo, $4(2 \%)$ tinham envolvimento da mão, e $3(1 \%)$ tinham envolvimento do punho ${ }^{20}$. Outros sítios nesta série com dois ou menos casos incluiu o ílio, ombro, costela, púbis, calcâneo, fêmur e articulação sacroilíaca.
Neste aspecto percebe-se que nosso sistema de notificação ainda é falho por não explicitar nem os sítios nem o grau de acometimento dos casos, o que nos limita nas comparações com a literatura internacional e nas correlações com a epidemiologia mundial da TBO. Dados anteriores a 2001 se apresentaram falhos, o que, juntamente com muitas variáveis em subnotificação, barra a análise pregressa da situação da TBO no país e nos impede de fazer um comparativo da evolução da entidade clínica no decorrer do tempo com maior fidelidade.

A TBEx é mais comum em pacientes com infecção pelo HIV, mas a TBO não é necessariamente aumentada em pacientes soropositivos para o HIV comparados a soronegativos $^{22}$, correlação esta também não encontrada em nossa epidemiologia, uma vez que, em 2017, os casos de TBO em HIV positivos correspondiam a apenas $15 \%$ do total. Porém há grande parte de pacientes com TBO que não são testados para HIV em nosso país, o que correspondia a $65,4 \%$ do total em 2006 e, em 2017, a $21 \%$ do total. Os dados sobre outras comorbidades relacionadas como a diabetes são deficientes tanto na literatura nacional como na mundial.

Observa-se que a prevalência de TB no Brasil ainda é grande e que, consequentemente, os casos de TBO também são prevalentes, compondo uma parcela significativa do espectro de acometimento da doença. Há destaque para os estados do Norte do país, com número crescente de casos. Com os dados obtidos da epidemiologia nacional, vemos que a principal faixa de acometimento da TBO é a população economicamente ativa, predominando homens entre a segunda e sexta década de vida moradores de áreas urbanas. Assim, vemos que os acometimentos osteoarticulares são importantes no contexto produtivo e de saúde para essa população e para o sistema de saúde nacional. Destaca-se, nesse contexto, a região Sudeste do país, que contribui com a maioria dos casos de TBO. Não obstante, essa região comporta a maior população, sendo também a mais economicamente desenvolvida, o que pode influenciar na quantidade de casos notificados devido a uma melhor qualidade tanto de diagnóstico quanto de registro.

A literatura nos mostra que, apesar de países desenvolvidos terem baixas taxas de TB, as ondas migratórias têm efeito impulsivo nos índices de TB, corroborando para que os casos de TBO ainda sejam mantidos no decorrer dos anos. O Brasil deve se atentar a este dado, pois recebeu grande aporte de imigrantes nos últimos anos de regiões com alta prevalência de TB. O acometimento da coluna é, na vasta maioria dos casos, o principal sítio independente da região geográfica analisada e, devido à alta morbidade, deve ser o principal sítio de TBEx na análise de qualquer contexto em que a TB seja abordada, sendo, dentro dos acometimentos extrapulmonares, a principal variável dentro da TBO. 


\section{CONCLUSÃO}

O sistema musculoesquelético permanece como um dos principais sítios de acometimento extrapulmonar da TB no Brasil. Nos países onde a tuberculose é endêmica, as crianças mais velhas e adultos jovens são mais comumente afetados com TBO como encontrado em nossa epidemiologia, enquanto nos países desenvolvidos a doença é frequentemente observada em idosos. A população mais acometida por essa doença no Brasil, no período analisado, consiste em uma população jovem que forma a força de trabalho.

Os homens continuam com maior acometimento conforme o sexo, e as condições socioeconômicas ainda são um fator relevante, pois a epidemiologia mundial aponta para essa disparidade. Há particularidades existentes relacionadas às realidades de cada região, devendo ser dado enfoque para a onda crescente de casos na região Norte do país. Comorbidades que podem influenciar o acometimento, como doenças crônicas como o diabetes e infecções como HIV, devem ser mais bem investigadas e abordadas.
Percebe-se que nosso sistema de notificação ainda é falho por não explicitar os sítios nem o grau de acometimento dos casos, o que limita comparações com a literatura internacional e correlações com a epidemiologia mundial da TBO. Dados anteriores a 2001 se apresentaram falhos, e, portanto, não foram objetos do estudo; o que, juntamente com muitas variáveis em subnotificação, barra a análise pregressa da situação da TBO no país e nos impede de fazer um comparativo da evolução da entidade clínica no decorrer do tempo com maior fidelidade.

As falhas observadas no sistema de notificação da TB no Brasil revelam a necessidade de melhoria nos mecanismos de registro. Nesse contexto, nosso estudo aponta para o aprimoramento das informações relativas a comorbidades, uso de drogas e discriminação de sítios anatômicos dos acometimentos extrapulmonares pela TB, em especial os osteomusculares. Com essas informações, seria possível melhorar as políticas públicas relativas às prevenções primária, secundária e terciária, uma vez que os grupos de risco e os casos de TBEx seriam melhor identificados.

Participação dos autores: Teles Filho $R V$ - Participou ativamente na concepção, delineamento, análise e interpretação dos dados, no manuscrito enviado. Abe GM - Participou na redação do manuscrito incluindo crítica intelectual importante de seu conteúdo. Azevêdo LHS - Participou na redação do manuscrito incluindo crítica intelectual importante de seu conteúdo. Melo NC - Participou na revisão do manuscrito incluindo crítica intelectual importante de seu conteúdo. Rabahi MF - Participou na revisão do manuscrito e na aprovação final da versão a ser publicada. Daher $M T$ - Participou na revisão do manuscrito e na aprovação final da versão a ser publicada.

\section{REFERÊNCIAS}

1. World Health Organization. Regional Office for South-East Asia Bending the curve - ending TB: Annual report 2017. India: World Health Organization, Regional Office for SouthEast Asia; 2017. Licence: CC BY-NC-SA 3.0 IGO. Available from: http://www.who.int/iris/handle/10665/254762.

2. Brasil. Ministério da Saúde. Secretaria de Vigilância em Saúde. Implantação do plano nacional pelo fim da tuberculose como problema de saúde pública no Brasil: primeiros passos rumo ao alcance das metas. Bol Epidemiol. 2018;49(11):1-18. Disponível em: https://portalarquivos2.saude.gov.br/images/ pdf/2018/marco/26/2018-009.pdf.

3. Brasil. Ministério da Saúde. Secretaria de Vigilância em Saúde, Departamento de Vigilância das Doenças Transmissíveis. Brasil Livre da tuberculose : plano nacional pelo fim da tuberculose como problema de saúde pública. Brasília; 2017. Disponível em: http://bvsms.saude.gov.br/bvs/ publicacoes/brasil_livre_tuberculose_plano_nacional.pdf.

4. Lopes AJ, Capone D, Mogami R, Tessarollo B, Leme D, Cunha $\mathrm{D}$, et al. Quais são os desafios para o diagnóstico da tuberculose extrapulmonar? Tuberculose extrapulmonar: aspectos clínicos e de imagem. Pulmão RJ. 2006;15(4):25361. Disponível em: http://www.sopterj.com.br/wp-content/ themes/_sopterj_redesign_2017/_educacao_continuada/ curso_tuberculose_5.pdf.

5. Garg RK, Somvanshi DS. Spinal tuberculosis: a review. J Spinal Cord Med. 2011;34(5):440-54. doi: 10.1179/2045772311Y.0000000023.
6. Kilborn T, van Rensburg PJ, Candy S. Pediatric and adult spinal tuberculosis. Neuroimaging Clin N Am. 2015;25(2):209-31. doi: 10.1016/j.nic.2015.01.002.

7. Esendagli-Yilmaz G, Uluoglu O. Pathologic basis of pyogenic, nonpyogenic, and other spondylitis and discitis. Neuroimaging Clin N Am. 2015;25(2):159-61. doi: 10.1016/j. nic.2015.01.011.

8. Dunn RN, Ben Husien M. Spinal tuberculosis. Bone Joint J. 2018;100-B(4):425-31. doi: 10.1302/0301-620X.100B4. BJJ-2017-1040.R1.

9. Souza PS, Puertas EB, Wajchenberg M, Eduardo C, Sousa A, Orto CCD. Tuberculose óssea na coluna vertebral : aspectos clínicos e cirúrgicos vertebral tuberculosis of the spine : clinical aspects and surgery. 2005;4(2):75-9.

10. Batirel A, Erdem H, Sengoz G, Pehlivanoglu F, Ramosaco E, Gülsün S, et al. The course of spinal tuberculosis (Pott disease): results of the multinational, multicentre Backbone-2 study. Clin Microbiol Infect. 2015;21(11):1008e9-1008e18. doi: 10.1016/j.cmi.2015.07.013.

11. Ledbetter LN, Salzman KL, Sanders RK, Shah LM. Spinal Neuroarthropathy: pathophysiology, clinical and imaging features, and differential diagnosis. RadioGraphics. 2016;36(3):783-99. doi: 10.1148/rg.2016150121.

12. Brasil. Ministério da Saúde. Departamento de Informática do Sistema Único de Saúde (DATASUS). Sistema e Informação de Agravos de Notificação (SINAN). Casos de tuberculose 
- desde 2001 [citado 14 set - 14 out. 2018]. Disponível em: http://www.datasus.gov.br/DATASUS/index.php?area $=02$ 03\&id=31009407\&VObj=http://tabnet.datasus.gov.br/cgi/ tabcgi.exe?sinannet/cnv/tuberc.

13. Centers for Disease Control and Prevention (CDC). Reported Tuberculosis in the United States, 2015. Atlanta, GA: US Department of Health and Human Services, CDC; 2016. Available from: https://www.cdc.gov/tb/statistics/ reports/2015/pdfs/2015_Surveillance_Report_FullReport. pdf.

14. Hodgson SP, Ormerod LP. Ten-year experience of bone and joint tuberculosis in Blackburn 1978-1987. J R Coll Surg Edinb. 1990;35(4):259-62.

15. Talbot J, Bismil Q, Saralaya D, Newton D, Frizzel R, Shaw D. Musculoskeletal tuberculosis in Bradford - a 6-year review. Ann R Coll Surg Engl. 2007;89(4):405-9. https://doi. org $/ 10.1308 / 003588407 X 183328$.

16. Colmenero JD, Jiménez-Mejías ME, Reguera JM, PalominoNicás J, Ruiz-Mesa JD, Márquez-Rivas J, et al. Tuberculous vertebral osteomyelitis in the new millennium: still a diagnostic and therapeutic challenge. Eur J Clin Microbiol Infect Dis. 2004;23(6):477-83. doi: 10.1007/s10096-0041148-y.
17. Ludwig B, Lazarus AA. Musculoskeletal tuberculosis. Dis Month. 2007;53(1):39-45. https://doi.org/10.1016/j. disamonth.2006.10.005.

18. Davidson PT, Horowitz I. Skeletal tuberculosis. A review with patient presentations and discussion. Am J Med. 1970;48(1):77-84. doi: 10.1016/0002-9343(70)90101-4.

19. Kim S-J, Kim JH. Late onset Mycobacterium tuberculosis infection after total knee arthroplasty: A systematic review and pooled analysis. Scand J Infect Dis. 2013;45(12):907-14. https://doi.org/10.3109/00365548.2013.830192.

20. Agarwal RP, Mohan N, Garg RK, Bajpai SK, Verma SK MY. Clinicosocial aspect of osteo-articular tuberculosis. J Indian Med Assoc. 1990;88:307-9.

21. Zhang X, Ji J, Liu B. Management of spinal tuberculosis: a systematic review and meta-analysis. J Int Med Res. 2013;41(5):1395-407. doi: 10.1177/0300060513498023.

22. Leibert E, Schluger NW, Bonk S, Rom WN. Spinal tuberculosis in patients with human immunodeficiency virus infection: clinical presentation, therapy and outcome. Tuber Lung Dis. 1996;77(4):329-34. https://doi.org/10.1016/S09628479(96)90097-0.

Recebido: 02.04.18

Aceito: 25.09 .19 